\title{
COMPORTAMENTO SEXUAL DE UNIVERSITÁRIAS DA ÁREA DA SAÚDE
}

\author{
SEXUAL BEHAVIOR OF HEALTH AREA UNIVERSITY STUDENTS
}

\author{
COMPORTAMIENTO SEXUAL DE UNIVERSITARIAS DEL ÁREA DE SALUD
}

Juliana Jordão Gomes ${ }^{1}$, Mylena Lucena Couto², Joyce Santos Nascimento ${ }^{3}$

\begin{abstract}
RESUMO
Objetivo: avaliar o comportamento de jovens universitárias da área da saúde em relação ao uso de métodos contraceptivos e à prevenção contra DST. Método: trata-se de um estudo transversal, analítico e quantitativo. Os dados foram coletados nos cursos de Enfermagem, Nutrição, Odontologia e Medicina do campus da Universidade Federal de Alagoas, em 2015, e contou com 279 estudantes. Resultados: do total, 68,5\% apresentavam vida sexual ativa; $73,8 \%$ relataram realizar sexo oral sem preservativo; 0 condon foi o método mais utilizado $(57,4 \%)$. Observou-se uma substituição da camisinha masculina pela pílula anticoncepcional com a idade. Foi encontrada associação estatística $(p=0,006)$ entre idade e realização de sexo vaginal sem preservativo de barreira. Conclusão: assim, as universitárias pesquisadas apresentam comportamento de risco para a gravidez e aquisição de DST, levando à suposição de que conhecimento sobre o tema não é suficiente para reduzir a exposição a práticas sexuais de risco.
\end{abstract}

\section{Descritores: Gravidez; Doenças Sexualmente Transmissíveis; Sexualidade; Estudantes.}

\section{ABSTRACT}

Objective: toevaluatethebehaviorofuniversitystudents in thehealtharea in relationtothe use ofcontraceptivemethodsandpreventionofSTDs. Method: it is a cross-sectional, analytical and quantitative study. The data was collected in the Nursing, Nutrition, Dentistry and Medicine courses of the campus of the Federal University of Alagoas in 2015, and relied on the participation of 279 students. Results: of the total, $68.5 \%$ had an active sexual life; $73.8 \%$ reported having oral sex without a condom; condon was the most used method (57.4\%). A substitution of the male condom by the contraceptive pill with age was observed. A statistical association $(p=0.006)$ was found between age and vaginal intercourse without barrier preservatives. Conclusion: thus, the university students surveyed present a risk behavior for pregnancy and STD acquisition, leading to the assumption that knowledge on the subject is not sufficient to reduce exposure to risky sexual practices.

1,2,3 Médicas. Universidade Federal de Alagoas/UFAL. Maceió (AL), Brasil.

Rev. Port. Saúde e Sociedade. 2019;4(1): 1006 - 1017. 


\section{RESUMEN}

Objetivo: evaluar el comportamiento de jóvenes universitarias del área de salud con respecto al uso de métodos contraceptivos y a la prevención de enfermedades de transmisión sexual. Método: se trata de un estudio transversal, analítico y cuantitativo. Los datos fueron recolectados en los cursos de Enfermería, Nutrición, Odontología y Medicina del campus de la Universidad Federal de Alagoas, en 2015, y asistieron 279 estudiantes. Resultados: el $68,5 \%$ de los encuestados tenía vida sexual activa; el $73,8 \%$ informó de la realización de sexo oral sin condón; el condón fue el método más utilizado $(57,4 \%)$. Se observó una sustitución del condón masculino para la píldora anticonceptiva con la edad. Fue encontrada asociación estadística $(p=0,006)$ entre edad y realización de sexo vaginal sin condón de barrera. Conclusión: los resultados muestran que las universitarias encuestadas presentan comportamiento de riesgo para el embarazo y adquisición de enfermedades de transmisión sexual, lo cual hace suponer que el conocimiento sobre el tema no es suficiente para reducir la exposición a las prácticas sexuales de riesgo.

\section{Descriptores: Embarazo; Enfermedades de Transmisión Sexual; Sexualidad; Estudiantes.}

\section{INTRODUÇÃO}

A Organização Pan-Americana de Saúde (OPAS) reconhece a juventude como o período dos 15 aos 24 anos, sendo um processo de transição, fundamentalmente sociológico, para que os jovens venham a assumir o papel social do adulto, do ponto de vista da família, da procriação e da profissão, com plenos direitos e suas devidas responsabilidades. ${ }^{1}$

A maior parte dos universitários brasileiros é constituída por jovens entre 17 e 24 anos, e o ingresso na universidade representa um momento marcante em suas vidas. O aluno recém-ingresso na universidade depara-se com situações novas, que suscitam sentimentos de alegria e excitação, além de insegurança e ansiedade. Nesse contexto, é comum que se iniciem comportamentos sexuais de risco, como a negligência ao uso de métodos contraceptivos e à prevenção contra Doenças Sexualmente Transmissíveis (DST)..$^{2-4}$

A gravidez precoce tem sido amplamente discutida por suas implicações biológicas, psicossociais e econômicas, além de estar associada à maior taxa de aborto espontâneo, a complicações de abortos inseguros e à maior morbimortalidade materna. A gravidez precoce indesejada contribui para o abandono dos estudos, a manutenção da pobreza e a estagnação da economia. Apesar de o homem também sofrer possíveis consequências do comportamento

Rev. Port. Saúde e Sociedade. 2019;4(1): 1006 - 1017. 
sexual e reprodutivo, os custos de uma gravidez geralmente são arcados pela mulher. ${ }^{5}$

Para as mulheres, os tempos modernos trouxeram maior liberdade sexual e autonomia em relação ao seu corpo e ao exercício de sua sexualidade, com a dissociação entre a necessidade de preservação da virgindade e o matrimônio, relação sexual e reprodução, amor e sexo. Ainda assim, as mulheres sofrem influência das relações tradicionais de gênero, que não foram totalmente quebradas. Alguns autores mostram que um papel mais ativo da mulher, em relação às suas vidas sexuais e ao próprio corpo, se faz importante também na prevenção de gestações indesejadas e infecções por DST. ${ }^{6-7}$

Uma sólida educação sexual está relacionada ao comportamento sexual e reprodutivo do ser humano, uma vez que os jovens que alcançam o mais alto nível educacional, em geral, adiam as uniões e as gestações para um futuro mais distante, quando alcançam certo sucesso profissional aliado à emancipação da família de origem. ${ }^{7-8}$

Apesar de haver, no meio acadêmico da área da saúde, maior acesso às informações acerca da sexualidade humana, bem como aos fatores de exposição às possíveis consequências de práticas sexuais inseguras, o estudo do perfil sexual da população universitária torna-se relevante em função do período de transição da referida população, no que diz respeito aos comportamentos social e sexual ${ }^{6}$. Alguns estudos têm mostrado que informação não implica necessariamente práticas sexuais seguras. ${ }^{4,9}$

É comum, ainda, a negligência aos preservativos de barreiras por meio da "negociação" com seus parceiros quanto à substituição da camisinha masculina baseada na fidelidade. Isso faz com que haja um aumento do não uso do preservativo durante o curso, o que torna as universitárias vulneráveis a infecções por DST.7,10

Além disso, o fato de a pesquisa se realizar com estudantes da área de saúde denota a importância no sentido de que este grupo lida diretamente com a saúde e o bem-estar do ser humano, em seus contextos acadêmico e profissional, supondo-se que, por isso, utilize esse conhecimento em favor de uma vida sexual segura no âmbito pessoal. Embora essas estudantes constituam uma pequena parcela da população jovem, ressalta-se seu papel como formadoras de opinião e propagadoras em potencial de uma vida saudável, o que as torna um grupo-chave na formulação de estratégias de educação em saúde. ${ }^{8}$

Rev. Port. Saúde e Sociedade. 2019;4(1): 1006 - 1017. 
Assim, considerando a carência de estudos que envolvam essa temática com o grupo em particular, este trabalho procura identificar a conduta sexual de jovens universitárias da área de saúde quanto ao risco de engravidar e às DST.

\section{MÉTODO}

Trata-se de um estudo transversal, analítico e quantitativo. Os dados foram coletados entre universitárias dos cursos da área da saúde do campus da Universidade Federal de Alagoas (UFAL) no município de Maceió, Alagoas, em 2016.

A amostra aleatória foi constituída por 279 estudantes do sexo feminino e o cálculo do tamanho da amostra foi realizado a partir da média percentual de estudantes do sexo feminino que ingressam nos cursos de Enfermagem, Nutrição, Odontologia e Medicina nos últimos anos. A coleta dos dados deu-se em sala de aula com as estudantes que concordaram em participar da pesquisa, após assinarem o Termo de Consentimento Livre e Esclarecido.

$O$ instrumento de pesquisa foi um questionário elaborado pelas pesquisadoras, após revisão de literatura, composto por perguntas fechadas com múltiplas alternativas. Foram estudados aspectos sociodemográficos, a vida sexual, o uso de preservativo, a antecedência de doenças sexualmente transmissíveis, a gravidez e o aborto.

Para a análise dos dados, foram utilizadas medidas estatísticas descritivas (médias, frequências e percentis) e analíticas para as categorias odds, por meio do programa Epi Info, versão 7.0. O estudo foi aprovado pelo Comitê de Ética em Pesquisa da Universidade Federal de Alagoas sob 0 protocolo no 30818114.1.0000.5013.

\section{RESULTADOS}

Foram estudadas 279 universitárias, estando a maioria na faixa etária dos 20 aos 22 anos de idade (40,9\%), afirmando-se predominantemente solteiras $(92,1 \%)$ e de religião católica $(58,8 \%)$. Referente à presença de parceiro sexual fixo, foi verificado que $60,0 \%$ das participantes afirmaram não o possuir. A maior parte da amostra afirmou ter apresentado entre um e três parceiros durante toda

Rev. Port. Saúde e Sociedade. 2019;4(1): 1006 - 1017. 
a vida sexual e 31,5\% não apresentavam vida sexual ativa no momento da pesquisa (Tabela 1 ).

Tabela 1. Distribuição das estudantes segundo a idade, o estado civil, a crença religiosa, o tipo e o número de parceiros sexuais.

\begin{tabular}{|c|c|c|}
\hline Idade & $n=279$ & $\%$ \\
\hline 17 a 19 anos & 55 & $19,7 \%$ \\
\hline 20 a 22 anos & 114 & $40,9 \%$ \\
\hline 23 a 25 anos & 76 & $27,2 \%$ \\
\hline 26 a 29 anos & 24 & $8,6 \%$ \\
\hline Acima de 30 anos & 10 & $3,6 \%$ \\
\hline \multicolumn{3}{|l|}{ Estado Civil } \\
\hline Solteira & 257 & $92,1 \%$ \\
\hline Casada & 14 & $5,0 \%$ \\
\hline Divorciada & 2 & $0,7 \%$ \\
\hline União consensual & 4 & $1,5 \%$ \\
\hline Ignorados & 2 & $0,7 \%$ \\
\hline \multicolumn{3}{|l|}{ Crença Religiosa } \\
\hline Católica & 164 & $58,8 \%$ \\
\hline Evangélica & 54 & $19,4 \%$ \\
\hline Sem religião & 34 & $12,2 \%$ \\
\hline Espírita & 16 & $5,7 \%$ \\
\hline Outros & 9 & $3,2 \%$ \\
\hline Ignorados & 2 & $0,7 \%$ \\
\hline \multicolumn{3}{|l|}{ Presença de parceiro fixo atual } \\
\hline Não & 167 & $59,9 \%$ \\
\hline Sim & 110 & $39,4 \%$ \\
\hline Ignorados & 2 & $0,7 \%$ \\
\hline \multicolumn{3}{|c|}{ Número de parceiros a partir do início da vida sexual } \\
\hline 1 a 3 & 166 & $59,5 \%$ \\
\hline Nenhum & 88 & $31,5 \%$ \\
\hline 4 a 6 & 14 & $5,0 \%$ \\
\hline Mais de 6 & 8 & $2,9 \%$ \\
\hline Ignorados & 3 & $1,1 \%$ \\
\hline
\end{tabular}

Das estudantes que afirmaram ter vida sexual ativa $(n=191), 82,2 \%$ informaram ter apresentado de um a três parceiros sexuais nos três meses anteriores à pesquisa (Tabela 2). Em relação à frequência das relações sexuais, a maior parte das entrevistadas $(55,5 \%)$ afirmou manter relações sexuais semanalmente e $16,8 \%(n=32)$, relações mensais.

Rev. Port. Saúde e Sociedade. 2019;4(1): 1006 - 1017. 
Tabela 2. Distribuição das estudantes sexualmente ativas segundo o número de parceiros sexuais e a frequência de relações sexuais nos últimos três meses.

\begin{tabular}{lcc}
\hline $\begin{array}{l}\text { Número de parceiros nos } \\
\text { últimos três meses }\end{array}$ & $\mathbf{n = 1 9 1}$ & $\%$ \\
\hline 1 a 3 & 157 & $82,2 \%$ \\
Nenhum & 33 & $17,3 \%$ \\
Ignorados & 1 & $0,5 \%$ \\
Frequência de relações & $n=191$ & $\%$ \\
sexuais nos últimos três & 106 & $55,5 \%$ \\
meses & 34 & $17,8 \%$ \\
Semanalmente & 32 & $16,8 \%$ \\
Nenhum & 17 & $8,9 \%$ \\
Mensalmente & 1 & $0,5 \%$ \\
Outros & 1 & $0,5 \%$ \\
Diariamente & & \\
Ignorados & & \\
\hline
\end{tabular}

Poucas universitárias afirmaram realizar sexo sem penetração - 76,4\% referiram raramente ou nunca o realizar. A tabela 3 mostra que o sexo oral sem preservativo de barreira é realizado pela maioria delas $(73,8 \%)$ e que o sexo vaginal sem preservativo também é realizado de forma expressiva, somando $68 \%$.

Tabela 3. Distribuição das estudantes segundo o tipo de relação sexual e a frequência de uso de preservativo (condom).

\begin{tabular}{|c|c|c|c|c|c|c|c|c|c|c|}
\hline 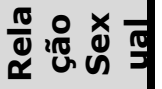 & & \multicolumn{3}{|c|}{$\begin{array}{c}\text { Com preservativo } \\
n=191(\%)\end{array}$} & & \multicolumn{4}{|c|}{$\begin{array}{c}\text { Sem preservativo } \\
\mathrm{n}=191(\%)\end{array}$} & \\
\hline 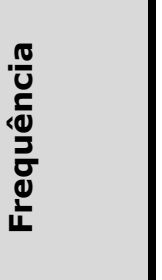 & $\begin{array}{l}\text { Uू } \\
\stackrel{5}{2}\end{array}$ & 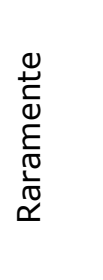 & 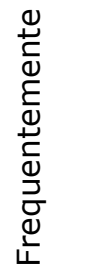 & 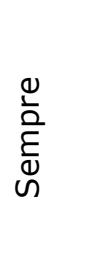 & $\begin{array}{l}0 \\
0 \\
\frac{0}{0} \\
\frac{0}{0} \\
\stackrel{0}{0} \\
0\end{array}$ & 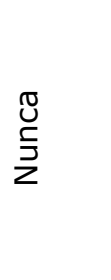 & 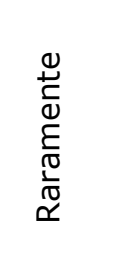 & 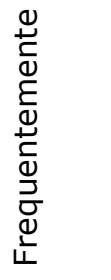 & 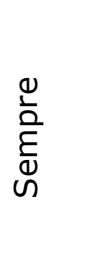 & 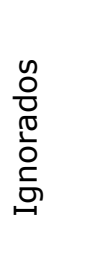 \\
\hline $\begin{array}{l}\overline{0} \\
\text { పे }\end{array}$ & 67,0 & 18,9 & 4,7 & 4,2 & 5,2 & 24,6 & 18,9 & 18,3 & 36,6 & 1,6 \\
\hline 首 & 17,3 & 23,0 & 29,3 & 26,7 & 3,7 & 29,3 & 25,6 & 22 & 20,4 & 2,6 \\
\hline
\end{tabular}

Rev. Port. Saúde e Sociedade. 2019;4(1): 1006 - 1017. 
Foram pesquisados os seguintes métodos contraceptivos: hormonais, de barreira e comportamental. A tabela 4 mostra que camisinha masculina (condomou preservativo de barreira) é o método mais utilizado em ambos os casos de situação quanto ao parceiro (48,6\% com parceiro fixo e $72,7 \%$ sem parceiro fixo). Entretanto, nota-se que as universitárias que possuem parceiros fixos usam relativamente menos preservativo de barreira e mais anticoncepcional hormonal se comparadas às que não possuem parceiro fixo. Informa-se que $38,1 \%$ afirmaram usar a pílula com parceiro sexual fixo e apenas 22,2\%, com parceiro temporário.

\section{Tabela 4. Caracterização dos métodos anticoncepcionais mais utilizados por tipo de parceiro.}

\begin{tabular}{lccccccc}
\hline \multirow{2}{*}{ MÉTODO } & \multicolumn{3}{c}{ PARCEIRO FIXO } & \multicolumn{2}{c}{ Total } \\
& \multicolumn{2}{c}{ SIM } & \multicolumn{2}{c}{ NÃO } & \multicolumn{2}{c}{} \\
& $\mathbf{n}$ & $\mathbf{\%}$ & $\mathbf{N}$ & $\mathbf{\%}$ & $\mathbf{n}$ & \% \\
\hline Hormonal & 66 & $38,1 \%$ & 22 & $22,2 \%$ & 88 & $32,4 \%$ \\
Camisinha masculina & 84 & $48,6 \%$ & 72 & $72,7 \%$ & 156 & $57,4 \%$ \\
Outros & 23 & $13,3 \%$ & 5 & $5,1 \%$ & 28 & $10,3 \%$ \\
\hline \multicolumn{2}{c}{ Total } & $\mathbf{1 7 3}$ & $\mathbf{1 0 0 , 0 \%}$ & $\mathbf{9 9}$ & $\mathbf{1 0 0} \%$ & $\mathbf{2 7 2}$ & $\mathbf{1 0 0 \%}$ \\
\hline
\end{tabular}

Realizaram-se cruzamentos entre uso de condom ou de anticoncepcional hormonal com ou sem parceiro fixo por idade como também correlacionando sexo vaginal com ou sem condom por idade. Foram estatisticamente significativas apenas as associações entre o uso de condom com parceiro fixo por idade (Odds=2,16; IC $95 \%=1,18-3,97 ; p=0,006)$ e sexo vaginal sem uso de condom por idade (Odds $\left.=2,27 ; \mathrm{IC}_{95 \%}=1,19-4,32 ; \mathrm{p}=0,006\right)$ (Tabela 5).

\section{Tabela 5. Relação entre idade das entrevistadas, condom (camisinha masculina), parceiro sexual fixo e sexo vaginal.}

\begin{tabular}{lcccc}
\hline \multicolumn{1}{c}{ Variável dependente } & $\begin{array}{c}\text { Variável } \\
\text { independente }\end{array}$ & OR & p & IC95\% \\
\hline Uso de condom com parceiro fixo & Idade & 2,16 & 0,006 & $\begin{array}{c}1,18- \\
3,97\end{array}$ \\
& Idade & 2,27 & 0,006 & $\begin{array}{c}1,19- \\
4,32\end{array}$ \\
\hline $\begin{array}{l}\text { Sexo vaginal sem uso de condom } \\
\text { (Nunca) }\end{array}$ & & & & \\
\hline
\end{tabular}

$\mathrm{OR}=$ Odds Ratio; $\mathrm{IC}_{95 \%=95 \%}$

Essa associação de dados mostrou que, entre as universitárias com parceiro sexual fixo, o uso de camisinha masculina foi mais expressivo nas participantes mais jovens. Quando avaliada a realização de sexo vaginal sem

Rev. Port. Saúde e Sociedade. 2019;4(1): 1006 - 1017. 
preservativo de barreira por idade, encontrou-se que as entrevistadas mais jovens responderam com mais frequência nunca realizar sem proteção, ou seja, relataram o uso consistente de condom com mais frequência.

Quando questionadas quanto à gravidez, a maioria das entrevistadas referiu nunca ter engravidado (94,9\%). Dentre as que informaram gravidez, $73,3 \%$ eram casadas ou estavam em união estável e 6,7\% referiram aborto espontâneo. Os métodos contraceptivos mais frequentemente utilizados quando engravidaram foram a "tabelinha" (36,4\%) e o "coito interrompido" (27,3\%).

\section{DISCUSSÃO}

Neste estudo, observou-se que a maior parte das entrevistadas possuía vida sexual ativa $(68,5 \%)$. Muitos adolescentes iniciam sua vida sexual próximo ao ingresso na universidade. A literatura mostra que esses dois eventos - o início da vida sexual e a entrada na universidade - representam marcos em direção à autonomia e à independência próprias da vida adulta. 3-4

Sabe-se que o número de parceiros sexuais é um fator que está relacionado com a vulnerabilidade às Doenças Sexualmente Transmissíveis. Este estudo evidencia que a maioria das estudantes $(59,0 \%)$ apresentou de um a três parceiros desde o início da vida sexual. Alguns autores demonstraram que a média de parceiros sexuais de estudantes da área de saúde durante toda a vida variava entre 3,07-3,42. ${ }^{2}$ Em estudo realizado com estudantes de Medicina, em 2011 , a atividade sexual com parceiro único foi relatada pela maioria $(72,5 \%)$ dos universitários. ${ }^{8}$

Quando questionadas quanto à frequência das relações sexuais, a maior parte das entrevistadas $(55,5 \%)$ afirmou apresentar relações sexuais semanalmente. Essa frequência na atividade sexual é apresentada de modo semelhante em um estudo que demonstra que $25,8 \%$ das estudantes, entre as que possuíam vida sexual ativa, tinham de 4-9 relações sexuais por mês. ${ }^{14}$

De acordo com os dados obtidos na pesquisa, a maioria das estudantes $(73,8 \%)$ realiza sexo oral sem preservativo de barreira. É reconhecido que essa modalidade de relação sexual também pode transmitir DST, o que torna esse resultado preocupante. Doenças como a sífilis são facilmente transmitidas pelo sexo oral por meio de ulcerações em região labial ou outras lacerações na região. O herpes labial pode, inclusive, causar o herpes genital devido ao sexo oral desprotegido. ${ }^{11}$

Rev. Port. Saúde e Sociedade. 2019;4(1): 1006 - 1017. 
Os dados acima referidos revelam um comportamento de risco da amostra pesquisada, apesar de todo o conhecimento adquirido durante os cursos da área da saúde. Em estudo realizado na mesma cidade do estudo relatado, foi demonstrado que, embora exista conhecimento entre a juventude acerca dos métodos contraceptivos, ainda há uma incapacidade de usá-los corretamente, uma realidade que a expõe aos riscos de aquisição de DST e a gravidezes não planejadas. $^{9}$

No tocante aos métodos contraceptivos, a camisinha masculina foi o método mais utilizado pelas entrevistadas, tanto com parceiro fixo $(48,6 \%)$ quanto com temporário $(72,7 \%)$. O preservativo de barreira é de fundamental importância não só na contracepção como também na prevenção das DST. A soberania da camisinha masculina também foi demonstrada em diversos estudos na literatura. ${ }^{7-9,12}$

Nota-se, também, que $68,0 \%$ das universitárias relataram não usar o preservativo de barreira (condom) em todas as relações sexuais vaginais. Também foi evidenciado que, apesar de o preservativo de barreira ser o método mais difundido entre a juventude, ainda há um elevado contingente de jovens que mantêm relações sexuais desprotegidas ou com uso ocasional do preservativo. ${ }^{12}$ De modo semelhante, este estudo alerta para um considerável uso inconsistente do preservativo em que o conhecimento adquirido durante a universidade não é posto em prática na vida sexual das pesquisadas.

Os resultados obtidos mostram também que o anticoncepcional hormonal, segundo método contraceptivo mais prevalente no estudo atual, é utilizado com mais frequência pelas participantes com parceiro sexual fixo. Esse achado está de acordo com os encontrados em outros estudos. ${ }^{7-8} \mathrm{O}$ advento desse método revolucionou a vida sexual da mulher, garantindo-lhe autonomia e possibilitando uma vida livre de riscos associados a uma gravidez não planejada. ${ }^{7}$

A diminuição da prevalência do uso da camisinha com parceiros fixos pode ter explicação no fato de relacionamentos estáveis trazerem maior confiança no parceiro, o que pode levar a uma despreocupação em relação à contaminação pelas DSTs e, consequentemente, ao abandono do uso do preservativo de barreira, único método contraceptivo capaz de realizar a dupla prevenção. 4,7-8,10

Estudos demonstram que o conhecimento sobre 0 parceiro e o desenvolvimento de uma relação estável, em geral, asseguram que a parceria sexual entre o casal é exclusiva. ${ }^{8}$ Desse modo, por desconsiderar que o parceiro

Rev. Port. Saúde e Sociedade. 2019;4(1): 1006 - 1017. 
possa ter sido exposto a outras situações de risco fora do contexto do relacionamento do casal, os casais com parcerias fixas inferem que não há risco de contrair DST, preocupando-se apenas com a prevenção contra a gravidez.

Observou-se, também, uma variação no padrão da contracepção no contexto de um relacionamento estável de acordo com a idade. Dados encontrados neste estudo revelam que, entre as universitárias com parceiro fixo, o uso de condom é mais frequente nas participantes mais jovens, entre 17 e 22 anos $(58,3 \%)$, sendo essa correlação estatisticamente significativa $(p=0,006)$.

Além disso, foi encontrada associação significativa $(p=0,006)$ entre idade e realização de sexo vaginal sem preservativo de barreira. As universitárias mais jovens afirmaram, com mais frequência, nunca realizar sexo vaginal sem preservativo $(38,9 \%)$, demonstrando o uso de condom com maior frequência. Assim, ser mais jovem é um fator de associação com o uso de condom, sendo protetor para aquelas com parceiro fixo (Odds=2,16; IC $95 \%=1,18-3,97$ ) e para a não realização de sexo vaginal sem preservativo (Odds=2,27; IC 95\%= $1,19-4,32)$.

Essas associações podem ser justificadas pela substituição do método contraceptivo com a idade. Estudos mostram que o preservativo de barreira, método mais utilizado no início da vida sexual ativa, tende a ser substituído por métodos hormonais, principalmente no contexto de relacionamentos estáveis. Por conseguinte, o anticoncepcional hormonal vai ganhando destaque com a passar da idade e com a estabilidade dos relacionamentos. ${ }^{7-8,10}$

Com relação aos dados obtidos quanto à gravidez, encontrou-se que a maioria nunca engravidou (94,9\%), corroborando a literatura nacional estudada. Em estudo sobre práticas sexuais em universitários, valores semelhantes foram encontrados, apesar de incluírem também o sexo masculino em seu grupo de entrevistados. ${ }^{15}$ No entanto, nesse mesmo estudo, uma pequena porcentagem dos estudantes que engravidaram encontrava-se em relação consensual ou em matrimônio, o que contrasta com as 73,3\% identificadas neste estudo.

Já em relação ao aborto, ao se traçar um comparativo com dados nacionais, encontrou-se, neste estudo, uma taxa relativamente elevada de abortos espontâneos (6,7\%). No entanto, deve-se levar em consideração a limitação do estudo pelo número pequeno de entrevistadas que engravidaram e à proibição legal do aborto no Brasil. Em estudo semelhante, a taxa de aborto espontâneo no Brasil foi de 14\%, tendo as jovens entre 15 a 24 anos uma

Rev. Port. Saúde e Sociedade. 2019;4(1): 1006 - 1017. 
participação em apenas $8 \%$ destes. ${ }^{13} \mathrm{Em}$ outro estudo, realizado na cidade de Maceió, $26,7 \%$ da amostra constituída por jovens estudantes já haviam praticado o aborto, $5^{5}$ o que contrasta com este estudo, em que nenhuma das participantes referiu ter abortado de forma intencional.

Apesar de a maioria das pesquisadas, de modo geral, fazer uso de métodos contraceptivos seguros, foi observado que, na parcela que engravidou, os métodos mais relatados foram a "tabelinha" e o "coito interrompido", alcançando $63,7 \%$ do total.

A "tabelinha" consiste em um método comportamental que visa a evitar as relações sexuais durante o período fértil da mulher, estando associada a uma falha de 14 a 47\%. Já o "coito interrompido" é praticado quando o homem retira o pênis antes da ejaculação e também possui um índice de falha alto (3-12\%), sendo considerado um método pouco seguro, pois depende do controle masculino. Dessa forma, ambos os métodos possuem elevados riscos de falhar na prevenção da gravidez. ${ }^{14}$

\section{CONCLUSÃO}

Este estudo encontrou comportamento de risco na vida sexual das universitárias estudadas. Destacam-se o uso inconsistente de preservativo nos diferentes tipos de relações sexuais e a variação do método de acordo com a estabilidade da parceria e a idade.

A substituição do método de barreira pelo anticoncepcional hormonal demonstra que, apesar do conhecimento técnico, essas jovens constituem um grupo vulnerável à aquisição de DSTs. Assim, os resultados desta pesquisa mostram que o conhecimento pode não ser suficiente para a mudança de comportamento no sentido de reduzir práticas sexuais de risco.

Enfatiza-se que os dados apresentados são importantes para subsidiar novos estudos sobre os riscos de uma vida sexual insegura. Conclui-se, então, que, a despeito do alto nível intelectual, torna-se necessária a atenção ao público estudado com políticas públicas e medidas de assistência estudantil para atuar nesses aspectos. Espera-se que os dados obtidos possam propiciar uma reflexão e a busca por profissionais que sejam capazes de avaliar suas próprias ações e estabelecer condutas adequadas em suas futuras vivências profissionais.

Rev. Port. Saúde e Sociedade. 2019;4(1): 1006 - 1017. 


\section{REFERENCES}

1. Buskieviczo A. Comportamento sexual e uso de métodos contraceptivos entre estudantes universitários de Cascavel-PR [dissertation]. Cascavel: Faculdade de AssizGurgacz; 2012.

2. Dessunti EM, Reis AOA. Vulnerabilitytostd/aids amonghealthgraduatestudents: a comparativestudybetweenfreshmenandseniors. Ciênccuid saúde. 2012; 11(suplem.):274-83.Doi: http://dx.doi.org/10.4025/cienccuidsaude.v11i5.17738

3. Sant'anna MJC, Carvalho KAM,Passarelli MLB, Coates V. Sexual behaviorofyounguniversitystudents. Adolesc saúde [Internet]. 2008 July [cited 2018 Aug 10]; 5(2):52-6.Availablefrom: http://www.adolescenciaesaude.com/detalhe_artigo.asp?id=62\#

4. D'Amaral HB, Rosa LA, Wilken RO, Spindola T, Pimentel MRAR, Ferreira LEM. Sexual practicesofnursingundergraduatesandpreventionofsexuallytransmitteddiseases.
RevEnfermUERJ.
2015
July/Aug;
23
(4):494-500.

Doi:http://dx.doi.org/10.12957/reuerj.2015.16823

5. CorreiaDS, CavalcanteJC, EgitoEST, MaiaEMC. Practiceofabortionamong teenagers: a study in tenschoolsof Maceió (AL, Brazil). Ciênc Saúde Colet. 2011 May; 16(5):2469-76. Doi:http://dx.doi.org/10.1590/S1413-81232011000500016

6. Borges MR, Silveira RE, Santos AS, Lippi UG. Sexual behaviour among initial academic students. J res fundam care online. 2015 Apr/June; 7(2):2505-15. Doi: http://dx.doi.org/10.9789/2175-5361.2015.v7i2.2505-2515

7. Delatorre MZ, Dias ACG. Knowledgeandpracticesoncontraceptionamongcollegestudents. Rev SPAGESP. 2015; 16(1):60-73.Availablefrom: http://pepsic.bvsalud.org/pdf/rspagesp/v16n1/v16n1a06.pdf

8. Aragão JCS, Lopes CS, Bastos FI. Sexual behaviorof medical students in Rio de Janeiro, Brazil. Revbraseduc med. 2011 July/Sept; 35(3):334-40.Doi: http://dx.doi.org/10.1590/S0100-55022011000300006

9. Correia DS, Pontes AC, Cavalcante JC, Egito ES, Maia EM. Adolescents: contraceptive knowledge and use, a Brazilian Study. ScientWorld J. 2009 Jan; 9:37-45.Doi: 10.1100/tsw.2009.8.

10. Tronco CB, Dell'Aglio DD. Descriptionofadolescent sexual behavior: sexual initiationandgender. Gerais: RevInterinst Psicol. 2012 Dec; 5(2):254-69. Doi:http://pepsic.bvsalud.org/pdf/gerais/v5n2/v5n2a06.pdf

11. Ministério da Saúde (BR). Adolescentes e jovens para a educação entre pares: sexualidades e saúde reprodutiva [Internet]. Brasília: Ministério da Saúde; 2011 [cited 2018

Availablefrom:https://unesdoc.unesco.org/ark:/48223/pf0000221728

Sept08].

12. Silva LP, Camargo FC, Iwamoto HH. Sexual behavioroffreshmen in anundergraduatehealthsciencesprogramof a publicuniversity. Revenferm atenção saúde. 2014; 3(1):39-52. Availablefrom: http://seer.uftm.edu.br/revistaeletronica/index.php/enfer/article/view/929/661

Rev. Port. Saúde e Sociedade. 2019;4(1): 1006 - 1017. 
13. Cecatti JG, Guerra GVQL, Sousa MH, Menezes GMS. Abortion in Brazil: a demographic approach. RevBrasGinecol Obstet. 2010 Mar; 32(3):105-11. Doi:http://dx.doi.org/10.1590/S0100-72032010000300002

14. Moreira MRC, Santos JFFQ. Betweenmodernityandtradition: sexual initiationofuniversityteensoriginallyfrom Piauí. Esc Anna Nery RevEnferm. 2011 July/Sept; $\quad$ 15(3):558-66. Doi:http://dx.doi.org/10.1590/S141481452011000300017

15. Falcão Junior JSP, Lopes EM, Freitas LV, Rabelo STO, Pinhieto AKB, Ximenes LB. Profile and sexual practicalofcollegestudentsfromhealtharea. Esc Anna Nery RevEnferm. 2007 Mar; 11(1):58-65.Doi: http://dx.doi.org/10.1590/S141481452007000100008

16. Sampaio Filho FJL, Sousa PRM, Vieira NFC, Nóbrega MFB, Gubert FA, Pinheiro PNC. Perceptionofriskofschooladolescents in relationtoalcoholconsumptionand sexual behavior. Rev Gaúcha Enferm. 2010 Sept; 31(3):508-14. Doi: http://dx.doi.org/10.1590/S1983-14472010000300014

17. Custódio G, Schuelter-Trevisol F, Daisson TJ, Zappelini CEM. Comportamento sexual e fatores de risco para a ocorrência de gravidez, DST e HIV em estudantes do município de Ascurra (SC). ArqCatMed [Internet]. 2009 [cited 2018 Aug19]; 38:56-61. Availablefrom:http://www.acm.org.br/revista/pdf/artigos/626.pdf

Rev. Port. Saúde e Sociedade. 2019;4(1): 1006 - 1017. 